\title{
High Sensitivity Test Signatures for Unconventional Analog Circuit Test Paradigms
}

\author{
Suraj Sindia* and Vishwani D. Agrawal ${ }^{\dagger}$ \\ Department of Electrical and Computer Engineering \\ Auburn University, Auburn, AL 36849 \\ *Email:szs0063@tigermail.auburn.edu †Email: vagrawal@eng.auburn.edu
}

\begin{abstract}
A method of testing for parametric faults in analog circuits based on a polynomial representation of fault-free function of the circuit is presented. The response of the circuit under test (CUT) is estimated as a polynomial in the root mean square (RMS) magnitude of the applied input voltage at a relevant frequency or DC. The test then classifies the CUT as fault-free or faulty based upon a comparison of the estimated polynomial coefficients with those of the fault-free circuit. The test application needs very little augmentation of the circuit to make it testable as only output parameters are used for classification. The method is validated on an active elliptic filter and is shown to uncover parametric faults causing deviations as small as 5\% from nominal values. Fault diagnosis based upon sensitivity of polynomial coefficients at relevant frequencies is discussed. Another type of circuit signatures in the form of probability moments of the output when test input is random noise are also proposed. It is shown that the sensitivity of either signature can be enhanced by a newly proposed nonlinear $V$ transform. Finally, an adaptive test framework leveraging from these signatures and the transform technique is shown to improve defect level and yield loss.
\end{abstract}

\section{INTRODUCTION}

Testing of linear circuits is well studied and several methods can be found in the literature [9], [15]-[17]. Guo and Savir [9] describe a scheme that is representative of coefficient based test schemes for analog circuits. The circuit under test (CUT) is subjected to frequency rich input signals and the output voltage alone is observed. With these input-output pairs one can estimate transfer function coefficients of the CUT under the assumption that it is a linear time-invariant (LTI) system. Next they compare these transfer function coefficient estimates with the ideal circuit transfer function coefficients, which are known a priori. The CUT is classified faulty if any of the estimated coefficients is outside a tolerance range. For example, the circuit shown in Figure 1 is a second order low pass filter and has a transfer function:

$$
H(s)=\frac{1}{\left(R_{1} R_{2} C_{1} C_{2}\right) s^{2}+\left(R_{1} C_{1}+\left(R_{1}+R_{2}\right) C_{2}\right) s+1}
$$

Clearly, the coefficients of the transfer function, $b_{0}=1, b_{1}=$ $\left(R_{1} C_{1}+\left(R_{1}+R_{2}\right) C_{2}\right), \quad b_{2}=R_{1} R_{2} C_{1} C_{2}$, are functions of circuit parameters $R_{1}, R_{2}, C_{1}, C_{2}$. Assuming single parametric faults, they find the minimum drift in any of the circuit component values that will cause the coefficients $b_{1}$ or $b_{2}$ ( $b_{0}$ here is a constant) to drift outside a tolerance range.

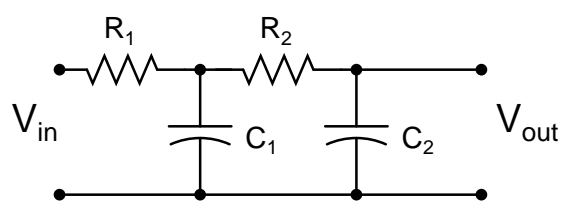

Fig. 1. Second order low pass filter.

However, this method [9] necessarily needs the CUT to be linear, as a frequency domain transfer function is possible only for an LTI system.

Several methods have been proposed for parametric fault testing of non-linear circuits [1], [2], [4]-[8], [13], [20]. To address the issue of parametric deviation, we would typically need more observables to have an idea about the parametric drift in circuit parameters. This would mean an increase in the complexity of the sensing circuit. However, we would also want minimal augmentation to tap any of the internal circuit nodes or currents. To overcome these seemingly contrasting requirements the method intended should have some way of "seeing through" the circuit with only the outputs and inputs at its disposal. References [9], [17] give such strategies for linear circuits as described earlier.

To extend this idea to general non-linear circuits we adopt a strategy where we express the function of the circuit as a polynomial using a Taylor series expansion [12] in terms of input voltage $v_{i n}$, about the point $v_{i n}=0$ as follows:

$$
\begin{aligned}
v_{\text {out }}=f\left(v_{\text {in }}\right)= & f(0)+\frac{f^{\prime}(0)}{n !} v_{\text {in }}+\frac{f^{\prime \prime}(0)}{2 !} v_{\text {in }}^{2}+\frac{f^{\prime \prime \prime}(0)}{3 !} v_{\text {in }}^{3}+ \\
& \cdots+\frac{f^{(n)}(0)}{n !} v_{\text {in }}^{n}+\cdots
\end{aligned}
$$

where $f(x)$ is a real function of $x$.

This method is very general as any analog circuit can be tested using this model. The technique applies equally well to linear circuits, which are a subclass of the general nonlinear circuits considered in this paper (originally appearing in [30], [31]). The accuracy, resolution and observability of faults uncovered depends on the degree of the polynomial expansion used in practice. Ignoring the higher order terms in (2), we can expand $v_{\text {out }}$ up to some $n$th power of $v_{i n}$, which gives us the approximation in (3). In order to increase the available observables to better track down parametric faults 
we can expand $v_{\text {out }}$ at multiple frequencies. Thus, we will have $m \times(n+1)$ observables where $m$ is the number of tones (frequencies) including DC at which $v_{\text {out }}$ is expanded and $n$ is the degree of expansion [10]:

$$
v_{\text {out }}=a_{0}+a_{1} v_{\text {in }}+a_{2} v_{i n}^{2}+\cdots+a_{n} v_{i n}^{n}
$$

where $a_{0}, a_{1}, a_{2}, \ldots, a_{n}$ are all real functions of circuit parameters $p_{k} \forall k$.

The special case of DC test that detects a subset of faults, was given in a recent paper [30]. Further, we assume that normal parameter variations (normal drift) in a good circuit are within a fraction $\alpha$ of their nominal value, where $\alpha<<1$. That is, every parameter $p_{k}$ is allowed to vary within the hypercube $p_{k, \text { nom }}(1-\alpha)<p_{k}<p_{k, \text { nom }}(1+\alpha) \forall k$, where $p_{k, \text { nom }}$ is the nominal value of parameter $p_{k}$. Whenever one or more coefficient values slip outside this hypercube we get a different set of coefficients reflecting a detectable fault. Therefore, equation (4) describes the hypercube for all parameters that correspond to either good machine values or undetectable parametric faults [4], [9], [20]:

$$
a_{i, \min }<a_{i}<a_{i, \max } \quad \forall i, \quad 0 \leq i \leq n
$$

This paper is organized as follows. Section II analyzes the coefficients of the polynomial expansion of the function $f\left(v_{i n}\right)$ and determines the detectable fault sizes of parameters. In Section III, we illustrate the testing problem being solved and discuss the proposed solution with an example. In Section IV, we generalize the solution to an arbitrarily large circuit. Section V presents the simulation results for some standard circuits. Section VI outlines the method of fault diagnosis using the proposed method. Sections VII and VIII introduce another type of signature, namely, probability moments and sensitivity enhancement of signatures by V-transform, respectively, that are described in detail elsewhere [24], [27]. An adaptive framework that leverages on the proposed circuit signatures is discussed in IX. We conclude in Section X.

The material presented in the paper is based on the doctoral dissertation [21] of the first author, written under the guidance of the second author. This manuscript is an entry to the final round of the TTTC E. J. McCluskey Doctoral Thesis Contest at 2013 International Test Conference.

\section{PRELIMINARIES}

The coefficients $a_{i} \forall i \quad 0 \leq i \leq n$ are, in general, non-linear functions of circuit parameters $p_{k} \forall k$. The rationale behind using these coefficients as metrics in classifying CUT as faulty or fault-free is based on the dependence of the coefficients on circuit parameters.

\section{A. Analysis of Polynomial Coefficients}

We discuss several significant results, proofs of which can be found elsewhere [21].

Theorem 1. If coefficient $a_{i}$ is a monotonic function of all parameters, then $a_{i}$ takes its limiting (maximum and minimum) values when at least one or more of the parameters are at the boundaries of their individual hypercube.

Lemma 1. If coefficient $a_{i}$ is a non-monotonic function of one or more circuit parameters $p_{i}$, then $a_{i}$ can take its limiting values anywhere inside the hypercube enclosing the parameters.

From Theorem 1 and Lemma 1 it is clear that by exhaustively searching the space in the hypercube of each parameter we can get the maximum and minimum values of the polynomial coefficient. Typically this can be formulated as a non-linear optimization problem to find the maximum and minimum values of coefficient with constraints on parameters allowing only a normal drift.

Theorem 2. In polynomial expansion of non-linear analog circuit there exists at least one coefficient that is a monotonic function of all circuit parameters.

From Lemma 1 and Theorem 2 we find that circuit parameter deviations have a bearing on coefficients and monotonically varying coefficients can be used to detect parametric faults of the circuit parameters.

Theorem 3. A continuous non-monotonic function $f: \Re \rightarrow$ $\Re$ can be decomposed into piecewise monotonic functions as follows:

$$
\begin{gathered}
f(x)=f(x) u\left(x_{0}-x\right)+f(x)\left(u\left(x-x_{0}\right)-u\left(x-x_{1}\right)\right)+ \\
f(x)\left(u\left(x-x_{1}\right)-u\left(x-x_{2}\right)\right)+\cdots \\
+f(x)\left(u\left(x-x_{n-1}\right)-u\left(x-x_{n}\right)\right)
\end{gathered}
$$

where $x_{0}, x_{1}, \cdots x_{n}$ are all stationary points of $f(x)$ and $u(x)= \begin{cases}1 & \forall x \geq 0 \\ 0 & \forall x<0\end{cases}$

Using Theorem 3, we can express every polynomial coefficient as a monotonic function of circuit parameters and thus we can use every coefficient to track the drifts in circuit parameters.

\section{B. Definitions}

Definition 1. Minimum size detectable fault (MSDF), $\rho$, of a parameter is defined as the minimum fractional deviation of a circuit parameter from its nominal value for it to be detectable with all other parameters being held at their nominal values. The fractional deviation can be positive or negative and is named upside-MSDF (UMSDF) or downside$M S D F(D M S D F)$, accordingly.

Definition 2. Nearly-minimum size detectable fault (NMSDF), $\rho^{*}$, of a parameter is defined as some fractional deviation of the circuit parameter from its nominal value with all the other parameters being held at their nominal values that is close to its MSDF with an error, $\epsilon$ (infinitesimally small). That is,

$$
\epsilon=|\rho-\rho *| \quad \epsilon<<1
$$


NMSDF also has notions of upside and downside as in the case of MSDF. In equation (6), $\epsilon$ can be perceived as a coefficient of uncertainty about the MSDF of a parameter. Let $\psi$ be the set of all coefficient values spanned by the parameters while varying within their normal drifts, i.e.,

$$
\begin{gathered}
\psi=\left\{v_{0}, v_{1}, \cdots, v_{n} \mid v_{0} \in A_{0}, v_{1} \in A_{1}, \cdots, v_{n} \in A_{n}\right\} \\
\forall_{k} \quad p_{k, \text { nom }}(1-\alpha)<p_{k}<p_{k, n o m}(1+\alpha)
\end{gathered}
$$

Note that by Definitions 1 and 2, $\psi$ includes all possible values of coefficients that are not detectable. Any parametric fault inducing coefficient value outside this set $\psi$ will result in a detectable fault.

\section{PRoblem Description And SKetch of Solution}

We shall first give an illustrative example of calculation of limits for polynomial coefficients for a simple circuit using MOS transistors. We shall follow that up with MSDF values for the circuit parameters.

Example: Two stage amplifier. Consider the cascade amplifier shown in Figure 2. The output voltage $V_{\text {out }}$ in terms of input voltage results in a fourth degree polynomial equation as follows:

$$
v_{\text {out }}=a_{0}+a_{1} v_{\text {in }}+a_{2} v_{\text {in }}^{2}+a_{3} v_{\text {in }}^{3}+a_{4} v_{\text {in }}^{4}
$$

where the constants $a_{0}, a_{1}, a_{2}, a_{3}$ are defined symbolically for transistors $\mathrm{M} 1$ and $\mathrm{M} 2$ operating in the saturation region:

$$
\begin{aligned}
& a_{0}=V_{D D}-R_{2} K\left(\frac{W}{L}\right)_{2}\left\{\begin{array}{l}
\left(V_{D D}-V_{T}\right)^{2}+ \\
R_{1}^{2} K^{2}\left(\frac{W}{L}\right)_{1}^{2} V_{T}^{4}- \\
2\left(V_{D D}-V_{T}\right)_{1}\left(\frac{W}{L}\right)_{1} V_{T}^{2}
\end{array}\right\} \\
& a_{1}=R_{2} K\left(\frac{W}{L}\right)_{2}\left\{\begin{array}{l}
4 R_{1}^{2} K^{2}\left(\frac{W}{L}\right)_{1}^{2} V_{T}^{3} \\
+2\left(V_{D D}-V_{T}\right) R_{1} K\left(\frac{W}{L}\right)_{1} V_{T}
\end{array}\right\} \\
& a_{2}=R_{2} K\left(\frac{W}{L}\right)_{2}\left\{\begin{array}{l}
2\left(V_{D D}-V_{T}\right) R_{1} K\left(\frac{W}{L}\right)_{1} \\
-6 R_{1}^{2} K^{2}\left(\frac{W}{L}\right)_{1}^{2} V_{T}^{2}
\end{array}\right\} \\
& a_{3}=4 V_{T} K^{3}\left(\frac{W}{L}\right)_{1}^{2}\left(\frac{W}{L}\right)_{2}^{2} R_{1}^{2} R_{2} \\
& a_{4}=-K^{3}\left(\frac{W}{L}\right)_{1}^{2}\left(\frac{W}{L}\right)_{2}^{2} R_{1}^{2} R_{2}
\end{aligned}
$$

When we substitute the nominal values, $V_{D D}=1.2 \mathrm{~V}, V_{T}=$ $400 \mathrm{mV},\left(\frac{W}{L}\right)_{M 1}=\frac{1}{2}\left(\frac{W}{L}\right)_{M 2}=20$, and $K=100 \mu \mathrm{A} / \mathrm{V}^{2}$, we get the coefficients in terms of parameters $R_{1}$ and $R_{2}$, as

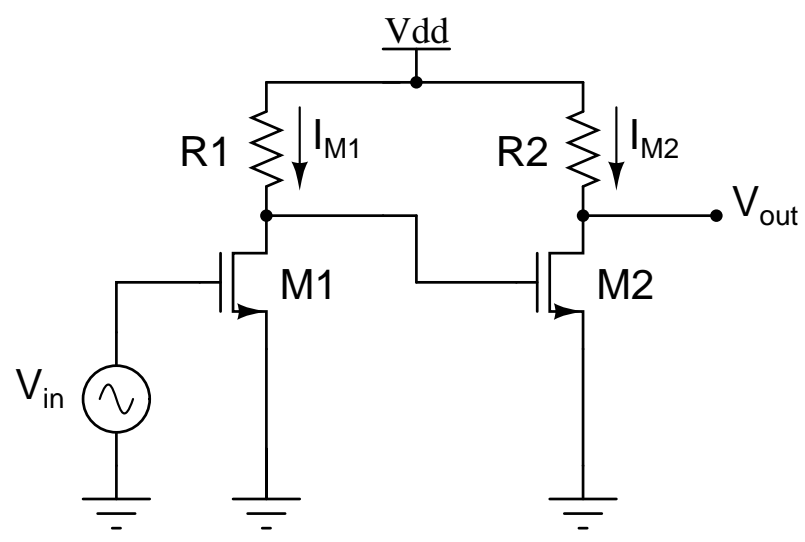

Fig. 2. Cascade amplifier.

follows:

$$
\begin{aligned}
& a_{0}=1.2-R_{2}\left(\begin{array}{l}
2.56 \times 10^{-3}+1.024 \times 10^{-7} R_{1}^{2} \\
-5.12 \times 10^{-4} R_{1}
\end{array}\right) \\
& a_{1}=4.096 \times 10^{-9} R_{1}^{2} R_{2}+5.12 \times 10^{-6} R_{1} R_{2} \\
& a_{2}=1.28 \times 10^{-5} R_{1} R_{2}-1.536 \times 10^{-8} R_{1}^{2} R_{2} \\
& a_{3}=2.56 \times 10^{-8} R_{1}^{2} R_{2} \\
& a_{4}=1.6 \times 10^{-8} R_{1}^{2} R_{2}
\end{aligned}
$$

To find the limiting values of the coefficient $a_{0}$ we assume the parameters $R_{1}$ and $R_{2}$ deviate by fractions $x$ and $y$ from their nominal values, respectively. For maximizing $a_{0}$ we have the objective function as given by (10), subject to constraints (11) through (15). Note that here we have set out to find MSDF of $R_{1}$. Similar approach can be used to find the MSDF of $R_{2}$.

$$
1.2-R_{2, \text { nom }}(1+y)\left\{\begin{array}{l}
2.56 \times 10^{-3}+ \\
1.024 \times 10^{-7} R_{1, \text { nom }}^{2}(1+x)^{2} \\
-5.12 \times 10^{-4} R_{1, \text { nom }}(1+x)
\end{array}\right\}
$$

$$
\begin{aligned}
& 4.096 \times 10^{-9} R_{1, \text { nom }}^{2}(1+x)^{2} R_{2, \text { nom }}(1+y) \\
& +5.12 \times 10^{-6} R_{1, \text { nom }}(1+x) R_{2, \text { nom }}(1+y) \\
& =4.096 \times 10^{-9} R_{1, \text { nom }}^{2}(1+\rho)^{2} R_{2, \text { nom }} \\
& +5.12 \times \quad 10^{-6} R_{1, \text { nom }}(1+\rho) R_{2, \text { nom }} \\
& 1.28 \times 10^{-5} R_{1, \text { nom }}(1+x) R_{2, \text { nom }}(1+y) \\
& -1.536 \times 10^{-8} R_{1, \text { nom }}^{2}(1+x)^{2} R_{2, \text { nom }}(1+y) \\
& =1.28 \times 10^{-5} R_{1, \text { nom }}(1+\rho) R_{2, \text { nom }} \\
& -1.536 \times 10^{-8} R_{1, \text { nom }}^{2}(1+\rho)^{2} R_{2, \text { nom }} \\
& 2.56 \times 10^{-8} R_{1, \text { nom }}^{2}(1+x)^{2} R_{2, \text { nom }}(1+y) \\
& =2.56 \times 10^{-8} R_{1, \text { nom }}^{2}(1+\rho)^{2} R_{2, \text { nom }} \\
& 1.6 \times 10^{-8} R_{1, \text { nom }}^{2}(1+x)^{2} R_{2, \text { nom }}(1+y) \\
& =1.6 \times 10^{-8} R_{1, \text { nom }}^{2}(1+\rho)^{2} R_{2, \text { nom }}
\end{aligned}
$$


TABLE I

MSDF FOR CASCADE AMPLIFIER OF FIGURE 2 WITH $\alpha=0.05$

\begin{tabular}{|c|c|c|}
\hline $\begin{array}{c}\text { Circuit } \\
\text { parameter }\end{array}$ & $\begin{array}{c}\text { \%upside } \\
\text { MSDF }\end{array}$ & $\begin{array}{c}\text { \%downside } \\
\text { MSDF }\end{array}$ \\
\hline \hline Resistor $R_{1}$ & 10.3 & 7.4 \\
\hline Resistor $R_{2}$ & 12.3 & 8.5 \\
\hline
\end{tabular}

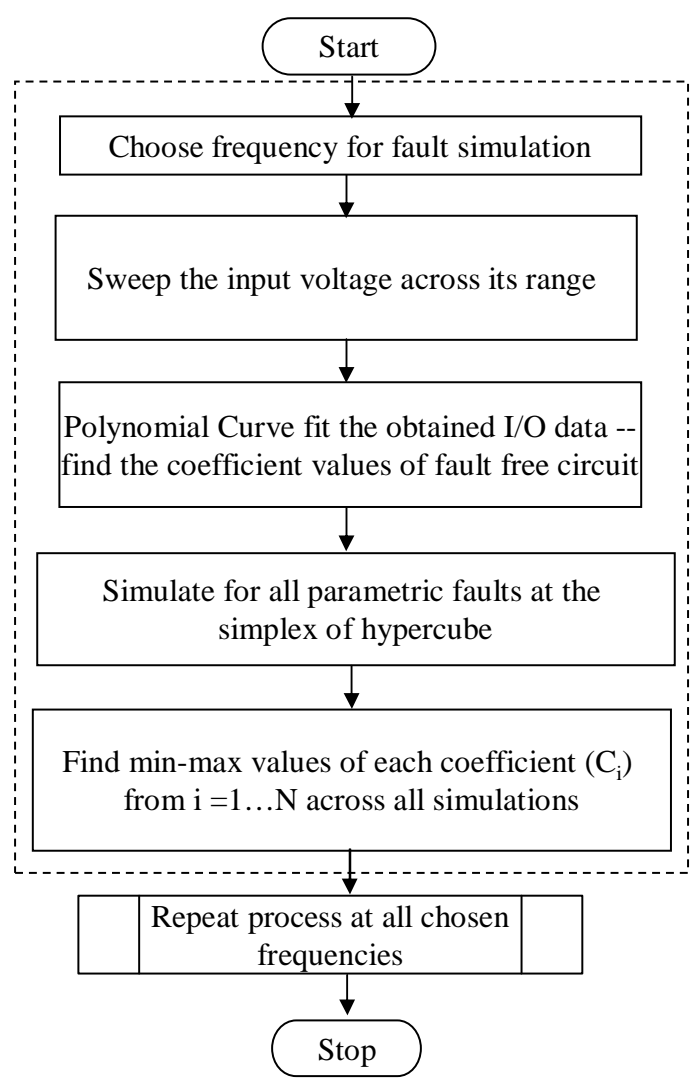

Fig. 3. Flow chart showing fault simulation process and bounding of coefficients.

$$
-\alpha \leq x, y \leq \alpha
$$

The extreme values for $x$ and $y$ on solving the above set of equations are obtained as, $x=-\alpha$ and $y=-\alpha$, giving us the MSDF value $\rho$ for $R_{1}$, as

$$
\rho=(1-\alpha)^{1.5}-1 \approx 1.5 \alpha-0.375 \alpha^{2}
$$

Table I gives the MSDF for $R_{1}$ and $R_{2}$ based on the above calculation.

\section{Generalization}

In general, the calculation as described above cannot be done for an arbitrarily large circuit. Such circuits are handled by obtaining a nominal numeric polynomial expansion of the fault-free circuit. This is done by sweeping the input voltage across all possible values and noting the corresponding output voltages using any of the standard circuit simulators like SPICE [19]. Now, the output voltage is plotted against the input voltage. A polynomial is fitted to this curve and the

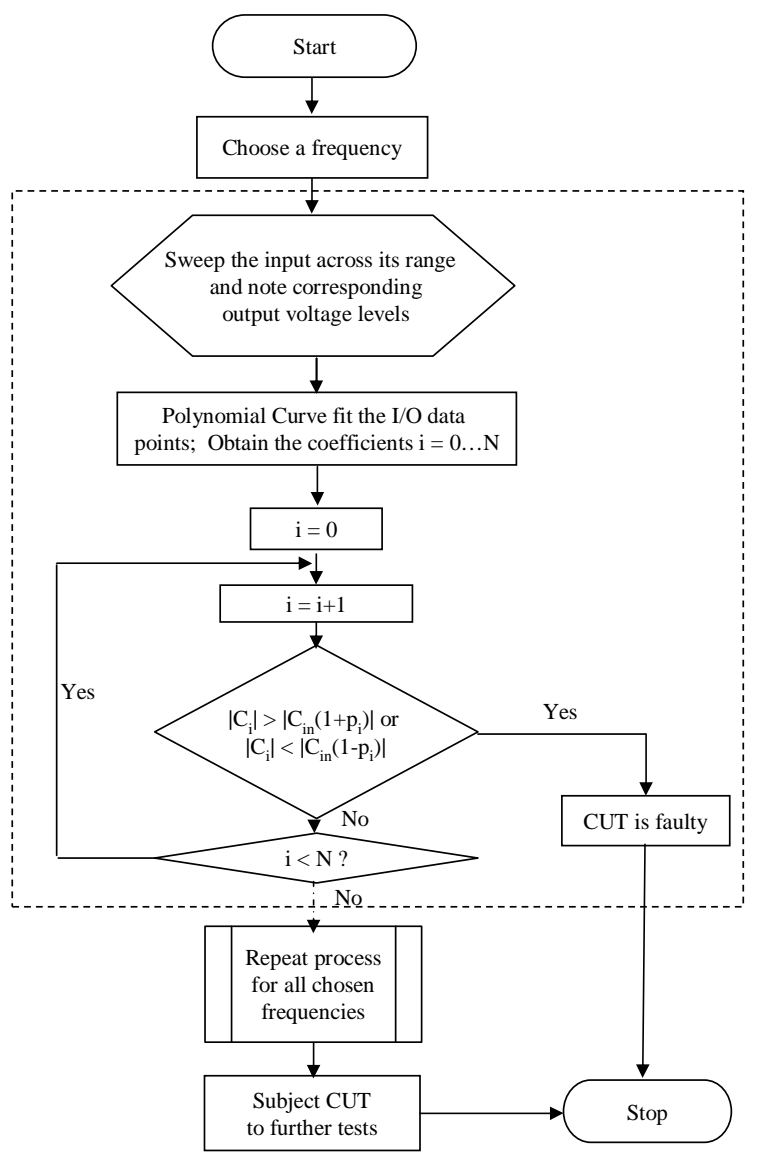

Fig. 4. Flow chart outlining test procedure for CUT.

coefficients of this polynomial are taken to be the nominal coefficients of the desired polynomial. The circuit is simulated for different drifts in the parameter values at equally spaced points from inside the hypercube enclosing each circuit parameter, spaced at a suitably chosen resolution $(=\epsilon)$. Polynomial coefficients are obtained for each of these simulations. The maximum and the minimum values of a coefficient in this search are taken as the limiting values on that coefficient. This process of modeling the circuit as a polynomial expansion and obtaining limit values on coefficients is repeated at "key" frequencies of interest. For example, the cut-off frequency in case of a non-linear filter can be a good candidate for such characterization. Once the limit values on all coefficients have been determined the CUT is subjected to full range of input at DC and each of the "key" frequencies. Its response to input sweep is curve fitted to a polynomial of order same as the fault-free circuit. If there are any coefficients that lay outside the limit values of corresponding coefficients of the fault-free circuit, we can conclude the CUT is faulty. The converse is also true with a high probability that is inversely proportional to coefficient of uncertainty $\epsilon$. Flow chart in Figure 3 summarizes the process of numerically finding the polynomial and finding the bounds on coefficients. Flow chart in Figure 4 outlines the procedure to test CUT using the described method. 


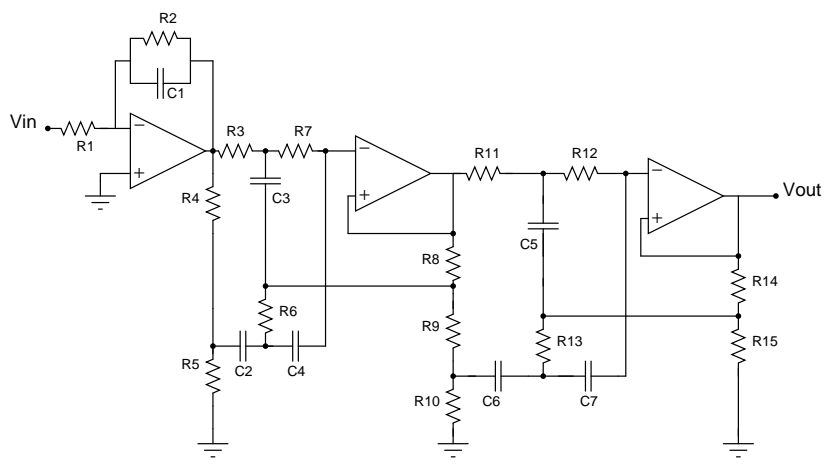

Fig. 5. Elliptic filter.

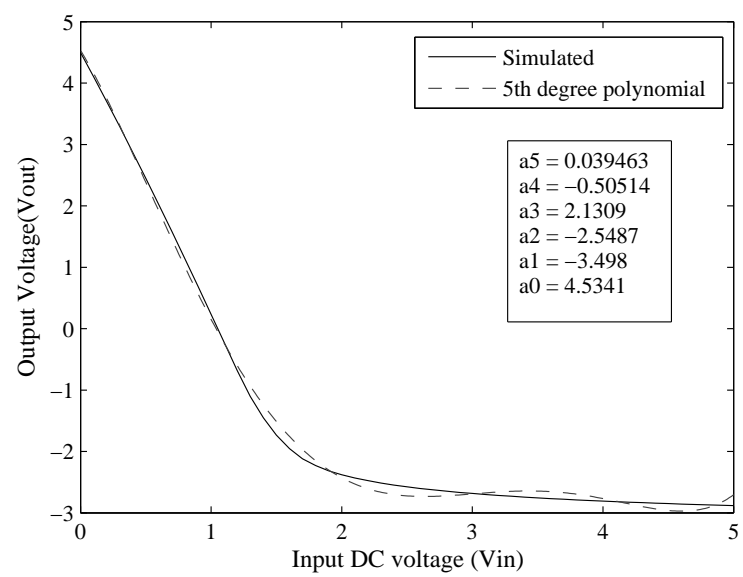

Fig. 6. DC response of elliptic filter with curve fitting polynomial.

\section{EXPERIMENTAL RESUlTS}

We subjected an elliptic filter shown in Figure 5 to polynomial coefficient based test [29]. The circuit parameter values are as in the benchmark circuit maintained by Stroud et al. [11]. We simulated the circuit at four different frequencies. Two of them were chosen close to its $3 \mathrm{~dB}$ cut-off frequency $\left(f_{c}\right)$, which is $1000 \mathrm{~Hz}$. The estimated polynomial expansion obtained by curve fitting the I/O plots at DC and the frequencies $f=100 \mathrm{~Hz}, 900 \mathrm{~Hz}, 1000 \mathrm{~Hz}, 1100 \mathrm{~Hz}$ are given by equations (17) through (21) and the corresponding plots tracing I/O response with polynomial are shown in Figures 6 through 10 . The combinations of parameter values leading to limits on the coefficients for the tone at $1000 \mathrm{~Hz}$ are shown in Table II. Further, the pass/fail detectability of several injected faults is tabulated in Table III.

$$
\begin{aligned}
v_{\text {out }}=4.5341- & 3.498 v_{\text {in }}-2.5487 v_{i n}^{2} \\
+ & 2.1309 v^{3} 3-0.50514 v_{i n}^{4}+0.039463 v_{\text {in }}^{5} \\
v_{\text {out }}=3 & +7.9 v_{\text {in }}-11 v_{\text {in }}^{2} \\
& +4.4 v_{\text {in }}^{3}-0.78 v_{\text {in }}^{4}+0.049 v_{\text {in }}^{5} \\
v_{\text {out }}= & 2.5+5.4 v_{\text {in }}-8.6 v_{\text {in }}^{2} \\
& +4 v_{\text {in }}^{3}-0.77 v_{\text {in }}^{4}+0.054 v_{\text {in }}^{5}
\end{aligned}
$$

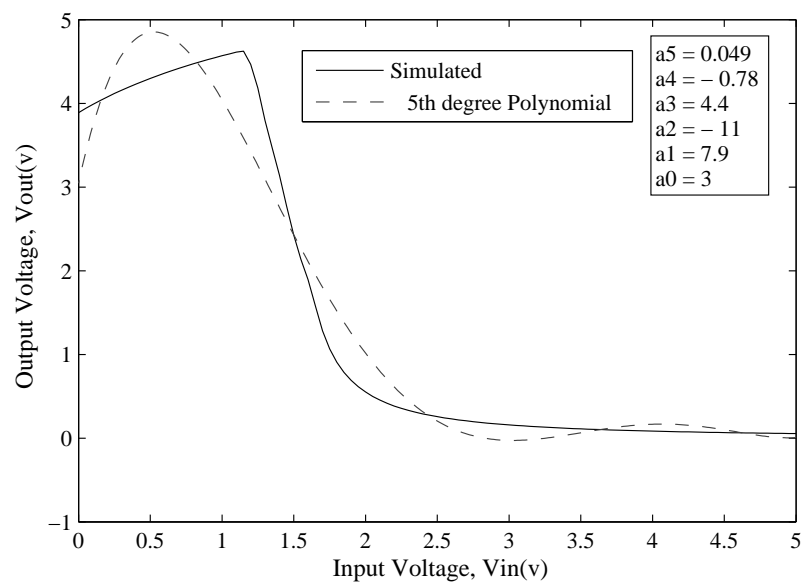

Fig. 7. Curve-fitting polynomial with coefficients at frequency $=100 \mathrm{~Hz}$.

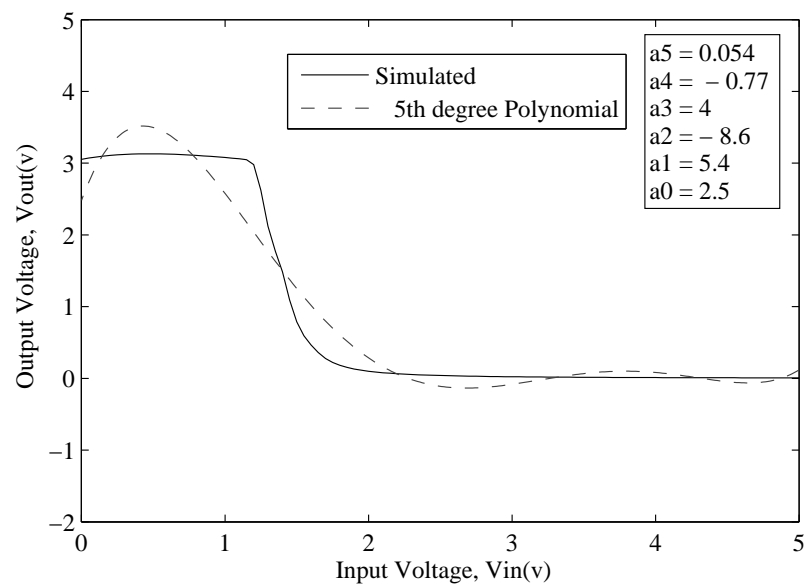

Fig. 8. Curve-fitting polynomial with coefficients at frequency $=900 \mathrm{~Hz}$.

$$
\begin{gathered}
v_{\text {out }}=1.1707+2.4132 v_{\text {in }}-3.8777 v_{\text {in }}^{2} \\
+1.8035 v_{\text {in }}^{3}-0.3465 v_{\text {in }}^{4}+0.023962 v_{\text {in }}^{5} \\
v_{\text {out }}= \\
\quad 0.23+0.48 v_{\text {in }}-0.74 v_{\text {in }}^{2} \\
\quad+0.34 v_{\text {in }}^{3}-0.063 v_{\text {in }}^{4}+0.0043 v_{\text {in }}^{5}
\end{gathered}
$$

\section{Fault Diagnosis}

Fault diagnosis using sensitivity of output to circuit parameters has been investigated in the literature [2], [34]. We have extended that approach exploiting the sensitivity of polynomial coefficients to circuit parameters [33]. The advantage of the new approach is an improved fault diagnosis without circuit augmentation. Sensitivity of $i^{\text {th }}$ coefficient $C_{i}$ to $k^{\text {th }}$ parameter $p_{k}$ is represented by $S_{p_{k}}^{C_{i}}$ and is given by:

$$
S_{P k}^{C i}=\frac{p_{k}}{C_{i}} \frac{\partial C_{i}}{\partial p_{k}}
$$


TABLE II

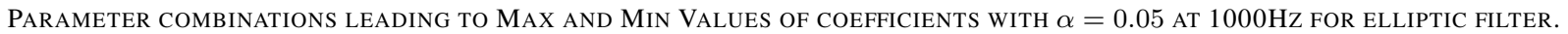

\begin{tabular}{|c|c|c|c|c|c|c|c|c|c|c|c|c|}
\hline \multicolumn{13}{|c|}{ Circuit Parameters (Resistance in $\Omega$, Capacitance in Farad) } \\
\hline \multirow{2}{*}{$\begin{array}{c}\text { Nominal } \\
\text { Values }\end{array}$} & \multicolumn{6}{|c|}{ Maximum values } & \multicolumn{6}{|c|}{ Minimum values } \\
\hline & $a_{0}$ & $a_{1}$ & $a_{2}$ & $a_{3}$ & $a_{4}$ & $a_{5}$ & $a_{0}$ & $a_{1}$ & $a_{2}$ & $a_{3}$ & $a_{4}$ & $a_{5}$ \\
\hline$R_{1}=19.6 \mathrm{k}$ & $18.6 \mathrm{k}$ & $18.6 \mathrm{k}$ & $20.5 \mathrm{k}$ & $20.5 \mathrm{k}$ & $20.5 \mathrm{k}$ & $18.6 \mathrm{k}$ & $18.6 \mathrm{k}$ & $18.6 \mathrm{k}$ & $18.6 \mathrm{k}$ & $18.6 \mathrm{k}$ & $20.5 \mathrm{k}$ & $20.5 \mathrm{k}$ \\
\hline$R_{2}=196 \mathrm{k}$ & $205 \mathrm{k}$ & $205 \mathrm{k}$ & $205 \mathrm{k}$ & $205 \mathrm{k}$ & $186 \mathrm{k}$ & $186 \mathrm{k}$ & $205 \mathrm{k}$ & $186 \mathrm{k}$ & $186 \mathrm{k}$ & $205 \mathrm{k}$ & $205 \mathrm{k}$ & $205 \mathrm{k}$ \\
\hline$R_{3}=147 \mathrm{k}$ & $139 \mathrm{k}$ & $139 \mathrm{k}$ & $154 \mathrm{k}$ & $139 \mathrm{k}$ & $139 \mathrm{k}$ & $139 \mathrm{k}$ & $139 \mathrm{k}$ & $139 \mathrm{k}$ & $154 \mathrm{k}$ & $139 \mathrm{k}$ & $139 \mathrm{k}$ & $139 \mathrm{k}$ \\
\hline$R_{4}=1 \mathrm{k}$ & 950 & 950 & $1.05 \mathrm{k}$ & $1.05 \mathrm{k}$ & $1.05 \mathrm{k}$ & $1.05 \mathrm{k}$ & $1.05 \mathrm{k}$ & 950 & $1.05 \mathrm{k}$ & 950 & 950 & $1.05 \mathrm{k}$ \\
\hline$R_{5}=71.5$ & 75 & 67 & 75 & 67 & 67 & 75 & 75 & 75 & 67 & 67 & 75 & 67 \\
\hline$R_{6}=37.4 \mathrm{k}$ & $35 \mathrm{k}$ & $39 \mathrm{k}$ & $39 \mathrm{k}$ & $35 \mathrm{k}$ & $35 \mathrm{k}$ & $39 \mathrm{k}$ & $39 \mathrm{k}$ & $39 \mathrm{k}$ & $35 \mathrm{k}$ & $35 \mathrm{k}$ & $35 \mathrm{k}$ & $35 \mathrm{k}$ \\
\hline$R_{7}=154 \mathrm{k}$ & $146 \mathrm{k}$ & $146 \mathrm{k}$ & $161 \mathrm{k}$ & $161 \mathrm{k}$ & $146 \mathrm{k}$ & $146 \mathrm{k}$ & $146 \mathrm{k}$ & $146 \mathrm{k}$ & $161 \mathrm{k}$ & $161 \mathrm{k}$ & $146 \mathrm{k}$ & $146 \mathrm{k}$ \\
\hline$R_{8}=260$ & 247 & 273 & 273 & 247 & 247 & 273 & 273 & 247 & 273 & 247 & 273 & 247 \\
\hline$R_{9}=740$ & 703 & 777 & 703 & 703 & 777 & 703 & 703 & 703 & 777 & 703 & 703 & 703 \\
\hline$R_{10}=500$ & 475 & 525 & 525 & 475 & 525 & 525 & 475 & 525 & 475 & 475 & 525 & 475 \\
\hline$R_{11}=110 \mathrm{k}$ & $115 \mathrm{k}$ & $115 \mathrm{k}$ & $115 \mathrm{k}$ & $104 \mathrm{k}$ & $104 \mathrm{k}$ & $104 \mathrm{k}$ & $115 \mathrm{k}$ & $115 \mathrm{k}$ & $104 \mathrm{k}$ & $115 \mathrm{k}$ & $104 \mathrm{k}$ & $104 \mathrm{k}$ \\
\hline$R_{12}=110 \mathrm{k}$ & $104 \mathrm{k}$ & $104 \mathrm{k}$ & $115 \mathrm{k}$ & $115 \mathrm{k}$ & $115 \mathrm{k}$ & $115 \mathrm{k}$ & $115 \mathrm{k}$ & $115 \mathrm{k}$ & $104 \mathrm{k}$ & $104 \mathrm{k}$ & $115 \mathrm{k}$ & $104 \mathrm{k}$ \\
\hline$R_{13}=27.4 \mathrm{k}$ & $28.7 \mathrm{k}$ & $26 \mathrm{k}$ & $26 \mathrm{k}$ & $26 \mathrm{k}$ & $28.7 \mathrm{k}$ & $28.7 \mathrm{k}$ & $26 \mathrm{k}$ & $26 \mathrm{k}$ & $28.7 \mathrm{k}$ & $26 \mathrm{k}$ & $28.7 \mathrm{k}$ & $26 \mathrm{k}$ \\
\hline$R_{14}=40$ & 42 & 38 & 42 & 38 & 38 & 42 & 42 & 38 & 42 & 42 & 38 & 42 \\
\hline$R_{15}=960$ & 912 & 912 & 912 & 912 & 912 & $1 \mathrm{k}$ & $1 \mathrm{k}$ & $1 \mathrm{k}$ & 912 & $1 \mathrm{k}$ & 912 & 912 \\
\hline$C_{1}=2.67 \mathrm{n}$ & $2.5 n$ & $2.5 n$ & $2.5 n$ & $2.5 n$ & $2.5 n$ & $2.5 n$ & $2.8 \mathrm{n}$ & $2.5 n$ & $2.8 \mathrm{n}$ & $2.8 \mathrm{n}$ & $2.8 n$ & $2.5 n$ \\
\hline$C_{2}=2.67 \mathrm{n}$ & $2.5 n$ & $2.8 n$ & $2.8 \mathrm{n}$ & $2.5 n$ & $2.8 n$ & $2.8 \mathrm{n}$ & $2.8 n$ & $2.8 n$ & $2.5 n$ & $2.8 \mathrm{n}$ & $2.5 n$ & $2.8 n$ \\
\hline$C_{3}=2.67 \mathrm{n}$ & $2.8 n$ & $2.8 \mathrm{n}$ & $2.8 \mathrm{n}$ & $2.5 n$ & $2.8 \mathrm{n}$ & $2.8 \mathrm{n}$ & $2.8 \mathrm{n}$ & $2.8 \mathrm{n}$ & $2.8 \mathrm{n}$ & $2.5 n$ & $2.8 n$ & $2.8 \mathrm{n}$ \\
\hline$C_{4}=2.67 \mathrm{n}$ & $2.5 n$ & $2.8 n$ & $2.5 n$ & $2.5 n$ & $2.5 n$ & $2.5 n$ & $2.5 n$ & $2.5 n$ & $2.8 \mathrm{n}$ & $2.5 n$ & $2.5 n$ & $2.8 n$ \\
\hline$C_{5}=2.67 \mathrm{n}$ & $2.5 n$ & $2.5 n$ & $2.5 n$ & $2.5 n$ & $2.5 n$ & $2.8 \mathrm{n}$ & $2.8 n$ & $2.8 n$ & $2.8 n$ & $2.8 \mathrm{n}$ & $2.8 n$ & $2.8 n$ \\
\hline$C_{6}=2.67 \mathrm{n}$ & $2.5 n$ & $2.8 \mathrm{n}$ & $2.5 n$ & $2.8 \mathrm{n}$ & $2.5 n$ & $2.8 \mathrm{n}$ & $2.5 n$ & $2.5 n$ & $2.8 \mathrm{n}$ & $2.8 \mathrm{n}$ & $2.8 \mathrm{n}$ & $2.5 n$ \\
\hline$C_{7}=2.67 \mathrm{n}$ & $2.5 n$ & $2.8 n$ & $2.8 n$ & $2.8 n$ & $2.8 \mathrm{n}$ & $2.5 n$ & $2.8 n$ & $2.5 n$ & $2.5 n$ & $2.5 n$ & $2.5 n$ & $2.8 n$ \\
\hline
\end{tabular}

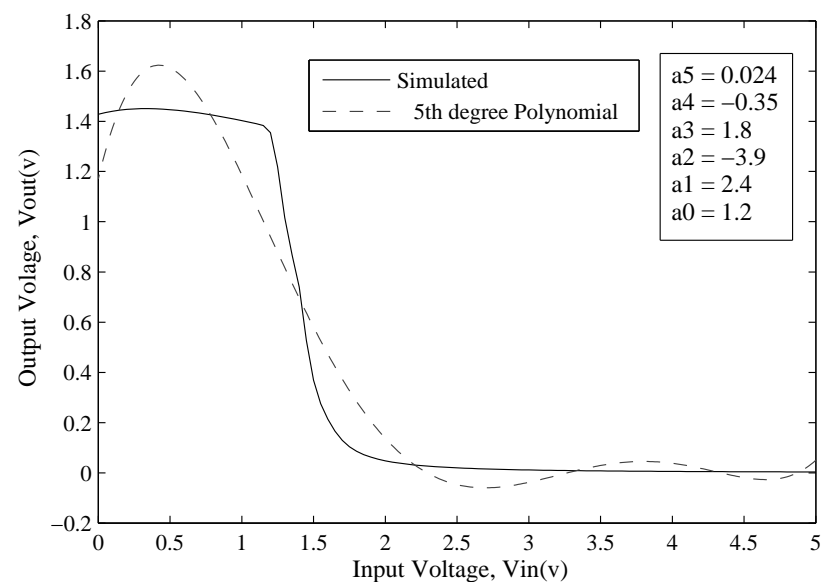

Fig. 9. Curve-fitting polynomial with coefficients at frequency $=1000 \mathrm{~Hz}$.

\section{A. Computation of Sensitivities}

Numerical computation of sensitivities given by (22) is accomplished by introducing fractional drifts $(=\alpha)$ in each component $\left(p_{k}, \forall k\right)$; simulating the circuit and measuring the fractional drift in each coefficient of the polynomial resulting from curve fitting operation. This way the numerical sensitivities are computed and a dictionary is maintained for sensitivities. The complexity in computation of sensitivities is linear in the number $N$ of circuit parameters, i.e., $O(N)$.

\section{B. Diagnosing Parametric Faults}

Restricting ourselves to single parametric faults, we find the descending order of sensitivities of coefficients (with respect to circuit parameter) that have exceeded their limiting values.

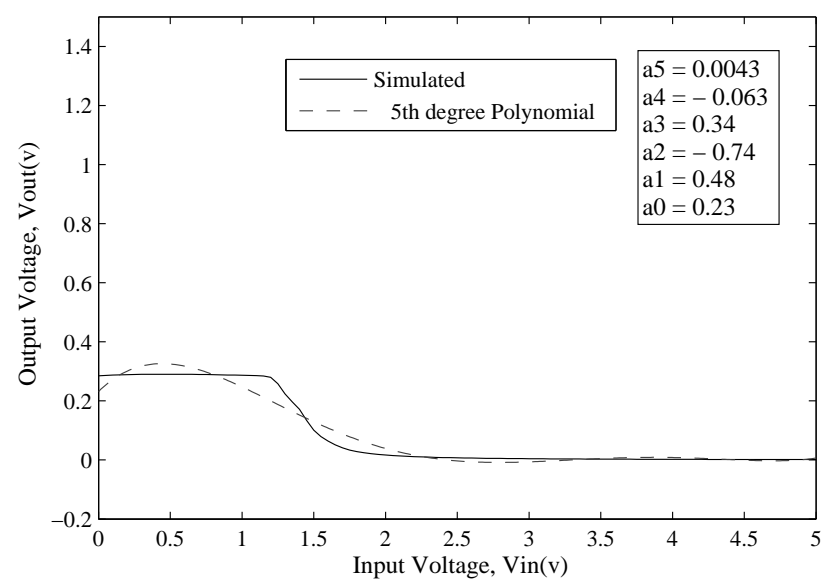

Fig. 10. Curve-fitting polynomial with coefficients at frequency $=1100 \mathrm{~Hz}$.

The parameter with highest sensitivity is said to be at fault with a probability $P\left(\delta p_{k} \mid \delta C_{i}\right)$ (which can be interpreted as the confidence in diagnosing fault), given by (23):

$$
P\left(\delta p_{k} \mid \delta C_{i)}=\phi\left(\frac{S_{P k}^{C i} \delta p_{k}}{\delta C_{i}}\right),\right.
$$

where $\delta p_{k}$ is the suspected drift in parameter $p_{k}, \delta C_{i}$ is the measured drift in coefficient, and $\phi($.$) is an appropriately$ chosen function that normalizes its argument into a valid probability number (between 0 and 1) [18].

\section{Fault Deduction}

At each frequency, the above process of diagnosis is repeated. This gives the set of fault sites above a certain 
TABLE III

DIAGNOSIS OF SOME INJECTED FAULTS IN ELLIPTIC FILTER AT VARIOUS FREQUENCIES.

\begin{tabular}{|c|c|c|c|c|c|c|}
\hline \multirow[t]{2}{*}{ Injected fault } & \multicolumn{5}{|c|}{ Coefficients out of Bounds at } & \multirow[t]{2}{*}{ Detected } \\
\hline & DC & $f_{1}=100 \mathrm{~Hz}$ & $f_{2}=900 \mathrm{~Hz}$ & $f_{3}=1000 \mathrm{~Hz}$ & $f_{4}=1100 \mathrm{~Hz}$ & \\
\hline$R_{1}$ down $15 \%$ & $a_{0}-a_{4}$ & $a_{1}-a_{4}$ & $a_{3}, a_{5}$ & $a_{2}, a_{4}$ & $a_{1}, a_{2}$ & Yes \\
\hline$R_{2}$ down $5 \%$ & $a_{2}, a_{5}$ & $a_{1}, a_{3}$ & $a_{1}, a_{5}$ & $a_{1}, a_{2}, a_{5}$ & $a_{1}, a_{2}$ & Yes \\
\hline$R_{3}$ up $10 \%$ & $a_{1}, a_{2}, a_{3}$ & $a_{3}, a_{5}$ & $a_{0}, a_{3}, a_{4}$ & $a_{1}, a_{3}, a_{4}$ & $a_{1}, a_{5}$ & Yes \\
\hline$R_{4}$ down $20 \%$ & $a_{0}-a_{3}$ & $a_{1}-a_{2}$ & $a_{2}, a_{3}$ & $a_{1}, a_{2}, a_{3}$ & $a_{2}, a_{3}$ & Yes \\
\hline$R_{5}$ up $15 \%$ & $a_{0}, a_{5}$ & $a_{1}$ & $a_{0}, a_{2}$ & $a_{0}, a_{2}, a_{3}$ & $a_{3}$ & Yes \\
\hline$R_{6}$ up 5\% & - & $a_{1}, a_{2}$ & $a_{2}, a_{3}, a_{5}$ & $a_{1}, a_{3}$ & $a_{1}$ & Yes \\
\hline$R_{7}$ down $10 \%$ & $a_{2}, a_{4}$ & $a_{3}, a_{5}$ & $a_{0}, a_{1}, a_{2}$ & $a_{1}, a_{4}, a_{5}$ & $a_{2}, a_{3}$ & Yes \\
\hline$R_{8}$ up $10 \%$ & - & $a_{2}$ & $a_{0}, a_{4}$ & $a_{0}, a_{2}, a_{5}$ & $a_{3}, a_{4}$ & Yes \\
\hline$R_{9}$ down $5 \%$ & - & $a_{3}, a_{2}$ & $a_{1}, a_{2}, a_{4}$ & $a_{2}, a_{3}, a_{5}$ & $a_{1}, a_{3}$ & Yes \\
\hline$R_{10}$ up $15 \%$ & - & $a_{1}, a_{4}$ & $a_{1}, a_{3}, a_{4}$ & $a_{0}, a_{1}, a_{4}$ & $a_{1}, a_{2}$ & Yes \\
\hline$R_{11}$ down $10 \%$ & $a_{0}, a_{2}$ & $a_{3}, a_{4}$ & $a_{0}, a_{1}$ & $a_{1}, a_{2}, a_{4}$ & $a_{1}, a_{2}$ & Yes \\
\hline$R_{12}$ down $15 \%$ & $a_{0}, a_{4}$ & $a_{1}, a_{3}$ & $a_{1}, a_{2}, a_{3}$ & $a_{1}, a_{2}$ & $a_{2}, a_{5}$ & Yes \\
\hline$R_{13}$ up $5 \%$ & - & $a_{3}, a_{5}$ & $a_{1}, a_{2}$ & $a_{1}, a_{2}, a_{4}$ & $a_{0}, a_{2}$ & Yes \\
\hline$R_{14}$ up $20 \%$ & - & $a_{1}, a_{3}$ & $a_{0}, a_{3}, a_{4}$ & $a_{0}, a_{1}, a_{2}$ & $a_{3}, a_{4}$ & Yes \\
\hline$R_{15}$ up $5 \%$ & - & $a_{4}$ & $a_{3}, a_{5}$ & $a_{0}, a_{1}, a_{3}$ & $a_{0}, a_{5}$ & Yes \\
\hline$C_{1}$ down $10 \%$ & - & $a_{4}, a_{5}$ & $a_{4}, a_{5}$ & $a_{1}, a_{2}, a_{3}$ & $a_{1}, a_{4}$ & Yes \\
\hline$C_{2}$ up $10 \%$ & - & $a_{2}, a_{3}$ & $a_{1}, a_{2}$ & $a_{2}, a_{3}, a_{4}$ & $a_{0}, a_{4}$ & Yes \\
\hline$C_{3}$ down $15 \%$ & - & $a_{1}, a_{3}$ & $a_{0}, a_{1}, a_{2}$ & $a_{4}, a_{5}$ & $a_{0}, a_{1}$ & Yes \\
\hline$C_{4}$ down $10 \%$ & - & $a_{0}, a_{1}$ & $a_{1}, a_{2}$ & $a_{2}, a_{3}$ & $a_{2}, a_{5}$ & Yes \\
\hline$C_{5}$ up 5\% & - & $a_{0}, a_{1}$ & $a_{1}, a_{5}$ & $a_{1}, a_{2}$ & $a_{3}, a_{4}$ & Yes \\
\hline$C_{6}$ up $15 \%$ & - & $a_{3}, a_{4}$ & $a_{1}, a_{2}, a_{4}$ & $a_{3}, a_{4}, a_{5}$ & $a_{1}, a_{2}$ & Yes \\
\hline$C_{7}$ up $15 \%$ & - & $a_{1}, a_{4}$ & $a_{1}, a_{3}, a_{4}$ & $a_{1}, a_{3}, a_{5}$ & $a_{3}, a_{4}$ & Yes \\
\hline
\end{tabular}

confidence level at each of these frequencies. The intersection of sets of fault sites at all the frequencies (and at DC) gives a fault site with much higher confidence level. That is, if the confidence of diagnosis of a fault site at one frequency is say $P_{i}$, then the resulting confidence level after diagnosis at all the frequencies is as follows [18]:

$$
P=1-\prod_{i=1}^{i=N}\left(1-P_{i}\right)
$$

where $N$ is the number of frequencies (including DC) at which the circuit is diagnosed.

Single parametric faults of the elliptic filter in Figure 5 were diagnosable with confidence levels up to $60 \%$ at each frequency. The resulting confidence level after fault deduction from the four frequencies at which it was diagnosed is about 98.9\%. The diagnosis results are given in Table IV for several injected single parametric faults. Another observation worthy of mention here is that the cardinality of set of fault sites detected at frequencies close to cut-off frequency is greater than that at frequencies closer to DC. This can be attributed to higher sensitivity of coefficients to circuit parameters at these frequencies. As a result, fault coverage is better by observing coefficient drifts at frequencies close to $f_{c}$. However these frequencies tend to be unfavorable for diagnosis as more than one parameter is likely to have displaced the coefficients out of their respective hypercubes. We can overcome this by looking at the set of fault sites obtained at frequencies much lower than $f_{c}(100 \mathrm{~Hz}$ used here).

\section{Probability Moments as a Circuit Signature}

The function of a circuit under test (CUT) is represented as a transformation on the probability density function of its input excitation, which is a continuous random variable (RV) with Gaussian probability distribution in [24], [25]. Probability moments of the output, now a transformed RV, are used as metrics for testing catastrophic and parametric faults in circuit components. The proposed use of probability moments as test metrics with white noise excitation as input addresses three important problems of analog circuit test, namely, 1) it reduces complexity of input signal design, 2) increases resolution of fault detection, and 3) reduces production test cost as it has no area overhead and marginally reduces test time. We also propose diagnosis of catastrophic faults in the circuit elements based on the unique relationship between specific moments of the output and circuit elements. We present a theoretical analysis, test and diagnosis procedures and SPICE simulation results for the proposed scheme applied to a benchmark elliptic filter and a low noise amplifier. We are able to detect all catastrophic faults and single faults in components that deviate from their nominal value by just over $10 \%$. We diagnose all catastrophic faults in the example circuits considered.

\section{V-TRAnsform as a Circuit Signature}

Parametric fault testing of non-linear analog circuits based on a new mathematical transform called V-transform is introduced in [23], [32]. V-transform acts on the polynomial expansion of the circuit's input-output voltage transfer function. It primarily serves to: 1) make the polynomial coefficients monotonic, 2) reduce masking of parametric faults 
TABLE IV

Parametric Fault Diagnosis With Confidence LeVels $\approx 98.9 \%$.

\begin{tabular}{|c|c|c|c|c|c|c|}
\hline \multirow[t]{2}{*}{ Injected fault } & \multicolumn{5}{|c|}{ Diagnosed fault sites at } & \multirow[t]{2}{*}{ Deduced fault site } \\
\hline & $\mathrm{DC}$ & $100 \mathrm{~Hz}$ & $900 \mathrm{~Hz}$ & $1000 \mathrm{~Hz}$ & $1100 \mathrm{~Hz}$ & \\
\hline$R_{1}$ down $15 \%$ & $R_{1}, R_{4}$ & $R_{1}$ & $R_{1}, R_{2}$ & $R_{1}, R_{2}, C_{1}$ & $R_{1}, C_{1}$ & $R_{1}$ \\
\hline$R_{2}$ down $5 \%$ & $R_{2}$ & $R_{2}, C_{1}$ & $R_{2}, R_{3}, C_{1}$ & $R_{2}, R_{3}$ & $R_{2}, C_{1}$ & $R_{2}$ \\
\hline$R_{3}$ up $10 \%$ & $R_{1}, R_{3}$ & $R_{3}, C_{3}$ & $R_{3}, R_{4}, C_{3}$ & $R_{3}$ & $R_{3}, C_{3}$ & $R_{3}$ \\
\hline$R_{4}$ down $20 \%$ & $R_{1}, R_{4}$ & $R_{1}, R_{4}$ & $R_{2}, R_{4}, C_{1}$ & $R_{1}, R_{2}, R_{4}$ & $R_{1}, R_{2}, R_{4}$ & $R_{4}$ \\
\hline$R_{5}$ up $15 \%$ & $R_{5}$ & $R_{5}, C_{2}$ & $R_{4}, R_{5}$ & $R_{4}, R_{5}, C_{2}$ & $R_{5}, R_{6}, C_{3}$ & $R_{5}$ \\
\hline$R_{6}$ up 5\% & - & $R_{6}, C_{2}$ & $R_{6}, R_{7}$ & $R_{6}, C_{2}, C_{4}$ & $R_{6}, C_{2}, C_{3}$ & $R_{6}$ \\
\hline$R_{7}$ down $10 \%$ & $R_{3}, R_{7}$ & $R_{7}, C_{3}$ & $R_{3}, R_{7}$ & $R_{3}, R_{6}, R_{7}$ & $R_{3}, R_{7}, C_{3}$ & $R_{7}$ \\
\hline$R_{8}$ up $10 \%$ & - & $R_{6}, R_{8}$ & $R_{8}, R_{9}$ & $R_{6}, R_{8}$ & $R_{8}, R_{9}$ & $R_{8}$ \\
\hline$R_{9}$ down $5 \%$ & - & $R_{8},=R_{9}$ & $R_{8}, R_{9}$ & $R_{9}, R_{10}$ & $R_{8}, R_{9}$ & $R_{9}$ \\
\hline$R_{10}$ up $15 \%$ & - & $R_{10}$ & $R_{10}, C_{6}$ & $R_{10}$ & $=R_{10}, C_{6}$ & $R_{10}$ \\
\hline$R_{11}$ down $10 \%$ & $R_{11}, R_{12}$ & $R_{11}$ & $R_{11}, C_{5}$ & $R_{11}, R_{12}$ & $R_{11}, R_{12}, C_{5}$ & $R_{11}$ \\
\hline$R_{12}$ down $15 \%$ & $R_{11}, R_{12}$ & $R_{11}, R_{12}$ & $R_{12}, C_{5}$ & $R_{12}, C_{5}$ & $R_{12}, C_{5}, C_{7}$ & $R_{12}$ \\
\hline$R_{13}$ up $5 \%$ & - & $R_{13}, C_{5}$ & $R_{13}, C_{7}$ & $R_{13}, C_{5}, C_{6}$ & $R_{13}, C_{5}$ & $R_{13}$ \\
\hline$R_{14}$ up $20 \%$ & - & $R_{14}$ & $R_{14}, R_{15}$ & $R_{14}, R_{15}$ & $R_{14}, R_{15}$ & $R_{14}$ \\
\hline$R_{15}$ up $5 \%$ & - & $R_{13}, R_{15}$ & $R_{14}, R_{15}$ & $R_{14}, R_{15}, C_{5}$ & $R_{14}, R_{15}$ & $R_{15}$ \\
\hline$C_{1}$ down $10 \%$ & - & $R_{2}, C_{1}$ & $R_{2}, C_{1}$ & $R_{2}, C_{1}$ & $R_{2}, C_{1}$ & $C_{1}$ \\
\hline$C_{2}$ up $10 \%$ & - & $R_{5}, C_{2}$ & $C_{2}, C_{4}$ & $C_{2}$ & $C_{2}$ & $C_{2}$ \\
\hline$C_{3}$ down $15 \%$ & - & $C_{3}$ & $R_{3}, C_{3}$ & $C_{3}$ & $C_{3}$ & $C_{3}$ \\
\hline$C_{4}$ down $10 \%$ & - & $R_{6}, C_{4}$ & $C_{2}, C_{4}$ & $C_{2}, C_{4}$ & $C_{2},=C_{4}$ & $C_{4}$ \\
\hline$C_{5}$ up $5 \%$ & - & $C_{5}$ & $R_{12}, C_{5}$ & $C_{5}$ & $C_{5}$ & $C_{5}$ \\
\hline$C_{6}$ up $15 \%$ & - & $R_{10}, C_{6}$ & $C_{6}, C_{7}$ & $C_{6}, C_{7}$ & $C_{6}, C_{7}$ & $C_{6}$ \\
\hline$C_{7}$ up $15 \%$ & - & $C_{6}, C_{7}$ & $C_{7}$ & $C_{6}, C_{7}$ & $C_{6}, C_{7}$ & $C_{7}$ \\
\hline
\end{tabular}

due to process variation, and 3) increase the sensitivity of polynomial coefficients to the circuit parameter variation, thus enhancing diagnostic resolution. There in it is shown that the sensitivity of V-transform coefficients (VTC) with respect to circuit parameter variation is up to 3 to 5 times greater than the sensitivity of polynomial coefficients. Fault diagnosis of parametric faults under process variation using VTC is also presented. We also propose a scheme to distinguish between circuit specifications failures due to process variation versus manufacturing defects which manifest as parametric faults in [22]. A complete description of the V-transform and its application on example circuits such as elliptic filter and low noise amplifier is presented in [23], [27]. Our work in [26], [28] computes a bound on the achievable defect level and fault coverage in coefficient based parametric fault test schemes such as the above.

\section{Adaptive Test With Signatures}

Signatures proposed in this thesis can be used in a closed loop framework such that the correlation of signatures to circuit specifications is further boosted up. Authors in [36] propose an adaptive test methodology for analog circuits in the alternate/signature test framework. Our preliminary studies on this approach have shown the feasibility of this approach with in conjunction with the signatures such as polynomial coefficients and V-transform coefficients proposed in the previous chapters.

\section{A. Overview}

Block diagram in Figure 11 shows the high-level conceptual framework of the adaptive test methodology using circuit

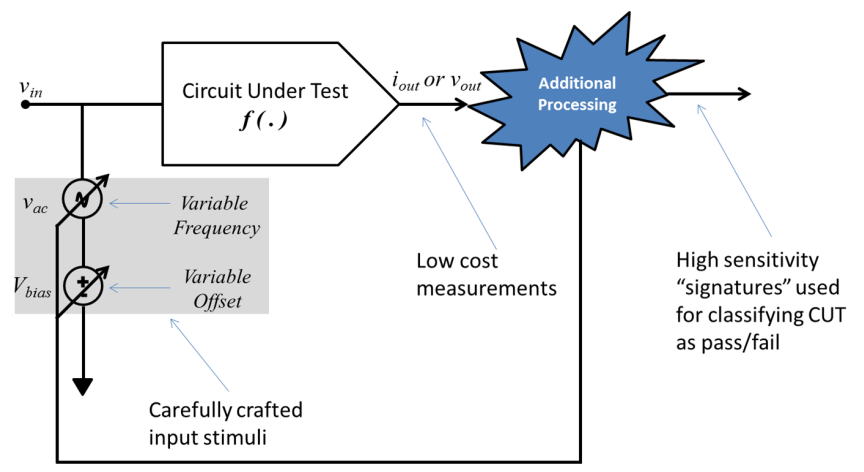

Fig. 11. Block diagram of the adaptive test system based on circuit signatures.

signatures. The circuit under test (CUT) is applied with a carefully crafted stimulus, whose output is then post-processed to generate the signatures such as polynomial coefficients or V-transform coefficients (proposed in previous chapters). The signatures are then used to compute correlation with the actual specification based on actual specification measurement of a small sampling of CUT at run-time. Based on the prevailing correlation, the input stimulus is tuned to achieve optimally sensitive signatures that has the highest degree of correlation to the circuit specification.

\section{B. Preliminary Experiments}

To our knowledge, a run-time, closed-loop tuning of the input stimulus to increase the correlation of the circuit signature to circuit specification for analog circuits has not been attempted before. Our initial experiments on a sample of 400 low noise amplifier [25], [27], [29] circuits show promising 


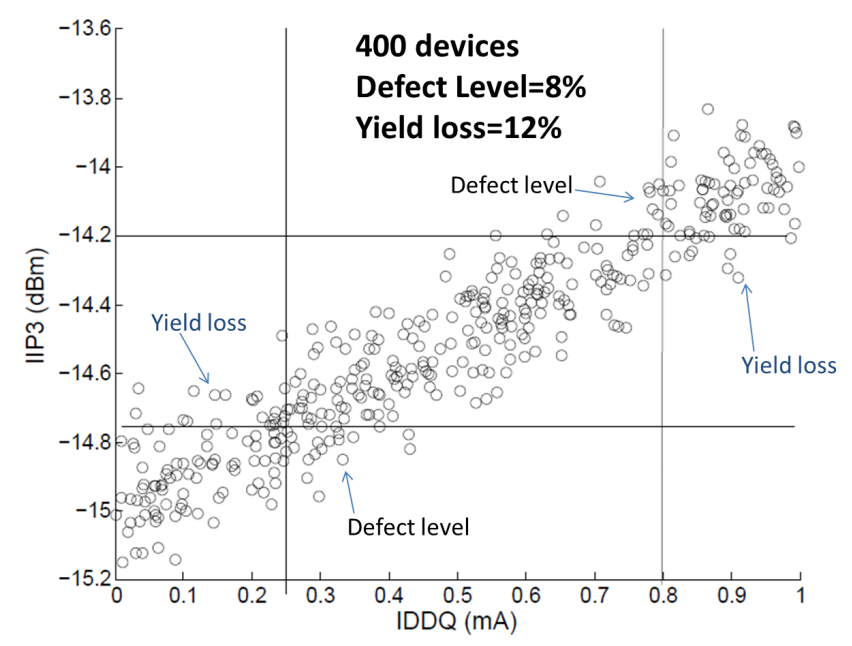

Fig. 12. Scatter plot of tested devices showing defect level and yield loss for the open loop signature test when the input stimulus is not tuned.

results on the possibility of using such closed-loop tuning on circuit stimulus to achieve high correlation with specification, which results in lower defect level and yield loss.

We compare Figures 12 and 13. Figure 13 shows the improved correlation between the signature, in this case, Vtransform of supply current $\left(I_{d d}\right)$, as opposed to just $I_{d d}$ and the specification IIP3 as shown in Figure 12. The penalty paid in this process is the extra test-time required to process the signatures and compute the required adjustments to the input stimulus at run-time (for example at production). However, it turns out that even minor adjustments in the input stimulus parameters can give rich dividends in the amount of correlation achieved through such closed-loop tuning. Furthermore, the computation time required for computing the change in stimulus along with the time required to initiate the change in the input stimulus amounts to about $10 \%$ increase in the total test-time when compared to test-flows that do not use such closed form tuning. Table $\mathrm{V}$ shows a comparison of three techniques, namely: testing for the specification "as is," using V-transform coefficients in open-loop, and using V-transform coefficients in closed-loop. Actual specification testing serves as the baseline case (or ideal scenario) for defect level (DL) and yield loss (YL). Notice that signatures taken in open-loop result in a DL and YL of $8 \%$ and $12 \%$ respectively. Having a closed-loop tuning of the stimulus improves DL and YL to $0.8 \%$ and $1.8 \%$ with a $5 \%$ time-penalty over the openloop case. But both these techniques give close to a $100 \mathrm{x}$ improvement in test-time over the baseline case (of measuring actual specification). More experiments are needed to study how this procedure would scale when the number of CUT are large and any other inadequacies of this approach.

\section{CONClusion}

A new approach for testing non-linear circuits based on polynomial expansion of the circuit function has been pro-

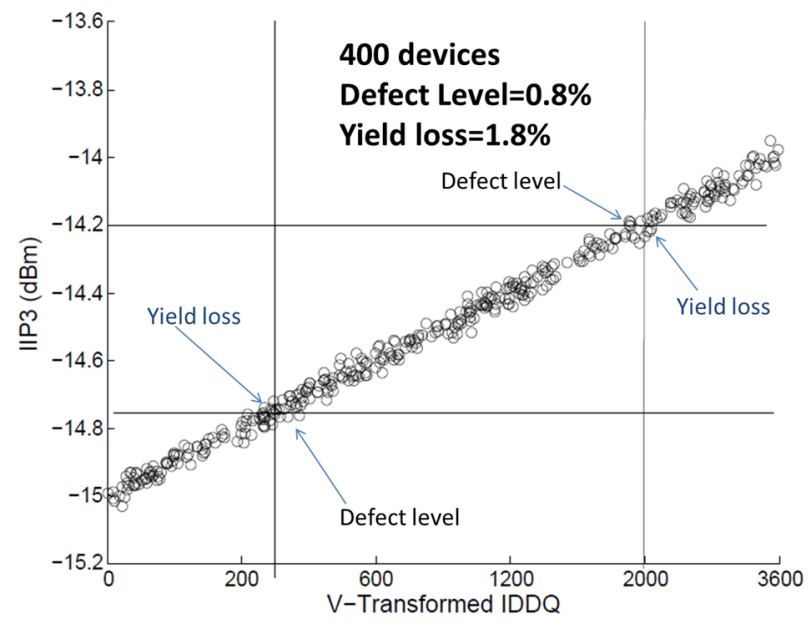

Fig. 13. Scatter plot of tested devices showing defect level and yield loss for the closed loop signature test when the input stimulus is adaptively tuned.

TABLE V

COMPARISON OF DEFECT LEVEL, YIELD LOSS, AND TEST TIME FOR ACTUAL SPECIFICATION TEST, SIGNATURE TEST IN OPEN LOOP, AND SIGNATURE TEST IN CLOSED LOOP.

\begin{tabular}{|c|c|c|c|}
\hline Test Method & Defect Level & Yield Loss & $\begin{array}{c}\text { Test Time } \\
\text { (per device) }\end{array}$ \\
\hline \hline Actual specification test & $0 \%$ & $0 \%$ & $15 \mathrm{~s}$ \\
\hline Open loop signature test & $8 \%$ & $12 \%$ & $100 \mathrm{~ms}$ \\
\hline Closed loop signature test & $0.8 \%$ & $1.8 \%$ & $105 \mathrm{~ms}$ \\
\hline
\end{tabular}

posed. Polynomial coefficients of the circuit function expanded at critical frequencies are capable of detecting and diagnosing circuit component faults as small as $5 \%$ for the example circuits considered. Circuit test signatures for increasing the component sensitivity to specification, namely V-transform; and simplifying input signal design effort, namely, probability moments are briefly discussed. Also, an adaptive framework for using these signatures and the consequent improvement in defect level and yield loss is demonstrated.

In proposing new signatures (polynomial coefficients and probability moments) and the sensitivity enhancement technique (V-transform) this research, on one hand, enhances the tool set of alternate test and, on the other hand, provides a bridge between the alternate [35]-[37] and model-based test [2] methods. In our future research, we plan to extend this bridge by combining specification-based tests [3], [14] and signatures together in the adaptive test framework proposed here for overall test cost minimization with given (acceptable) defect level and yield loss.

\section{ACKNOWLEDGMENT}

This research is supported in parts by the National Science Foundation Grants CCF-1116213 and IIP-0738088, and by the Wireless Engineering Research and Education Center at Auburn University. 


\section{REFERENCES}

[1] A. Abderrahman, E. Cerny, and B. Kaminska, "Optimization Based Multifrequency Test Generation for Analog Circuits," Journal of Electronic Testing: Theory and Applications, vol. 9, no. 1-2, pp. 59-73, Mar 1996.

[2] N. Ben Hamida and B. Kaminska, "Multiple Fault Analog Circuit Testing by Sensitivity Analysis," Journal of Electronic Testing: Theory and Applications, vol. 4, no. 4, pp. 331-343, Nov. 1993.

[3] M. L. Bushnell and V. D. Agrawal, Essentials of Electronic Testing for Digital, Memory and Mixed-Signal VLSI Circuits. Springer, 2000.

[4] S. Chakravarty and P. J. Thadikaran, Introduction to IDDQ Testing. Springer, 1997.

[5] S. Cherubal and A. Chatterjee, "Test Generation Based Diagnosis of Device Parameters for Analog Circuits," in Proc. Design, Automation and Test in Europe Conf., 2001, pp. 596-602.

[6] G. Devarayanadurg and M. Soma, "Analytical Fault Modeling and Static Test Generation for Analog ICs," in Proc. International Conf. Computer-Aided Design, Nov. 1994, pp. 44-47.

[7] S. L. Farchy, E. D. Gadzheva, L. H. Raykovska, and T. G. Kouyoumdjiev, "Nullator-Norator Approach to Analogue Circuit Diagnosis Using General-Purpose Analysis Programmes," International Journal of Circuit Theory and Applications, vol. 23, no. 6, pp. 571-585, Dec. 1995.

[8] R. K. Gulati and C. F. Hawkins, IDDQ Testing of VLSI Circuits. Springer, 1993.

[9] Z. Guo and J. Savir, "Analog Circuit Test Using Transfer Function Coefficient Estimates," in Proc. International Test Conf., Oct. 2003, pp. 1155-1163.

[10] A. Halder, S. Bhattacharya, and A. Chatterjee, "Automatic Multitone Alternate Test Generation for RF Circuits Using Behavioral Models," in Proc. International Test Conf., Nov. 2003, pp. 665-673.

[11] R. Kondagunturi, E. Bradley, K. Maggard, and C. Stroud, "Benchmark Circuits for Analog and Mixed-Signal Testing," in Proc. 20th International Conf. Microelectronics, Mar. 1999, pp. 217-220.

[12] E. Kreyzig, Advanced Engineering Mathematics. Wiley, 2005.

[13] W. L. Lindermeir, H. E. Graeb, and K. J. Antreich, "Analog Testing by Characteristic Observation Inference," IEEE Trans. Comp. Aided Design, vol. 23, no. 6, pp. 1353-1368, June 1999.

[14] M. Mahoney, DSP Based Testing of Analog and Mixed-Signal Circuits. IEEE Computer Society Press, 1987.

[15] L. Milor and V. Visvanathan, "Detection of Catastrophic Faults in Analog Integrated Circuits," IEEE Trans. Comp. Aided Design, vol. 8, no. 6, pp. 114-130, June 1989.

[16] N. Nagi, A. Chatterjee, A. Balivada, and J. A. Abraham, "FaultBased Automatic Test Generator for Linear Analog Devices," in Proc. International Conf. Computer Aided Design, May 1993, pp. 88-91.

[17] V. Panic, D. Milovanovic, P. Petkovic, and V. Litovski, "Fault Location in Passive Analog RC Circuits by Measuring Impulse Response," in Proc. 20th International Conf. Microelectronics, Sept. 1995 , pp. 12-14.

[18] A. Papoulis, Probability, Random Variables, and Stochastic Processes. McGraw-Hill, 1965.

[19] T. L. Quarles, D. O. Pederson, A. R. Newton, A. L. Sangiovanni-Vincentelli, C. Wayne, and J. M. Rabaey, "The Spice Page," accessed June 23, 2013. http://bwrcs.eecs.berkeley.edu/Classes/IcBook/SPICE/.

[20] R. Rajsuman, IDDQ Testing for CMOS VLSI. Artech House, 1995.
[21] S. Sindia, High Sensitivity Signatures for Test and Diagnosis of Analog, Mixed-Signal and Radio-Frequency Circuits. $\mathrm{PhD}$ thesis, Auburn University, Alabama, USA, Aug. 2013.

[22] S. Sindia, V. D. Agrawal, and V. Singh, "Distinguishing Process Variation Induced Faults from Manufacturing Defects in Analog Circuits using V-Transform Coefficients," in Proc. 43rd IEEE Southeastern Symp. System Theory, Mar. 2011, pp. 231-236.

[23] S. Sindia, V. D. Agrawal, and V. Singh, "Non-Linear Analog Circuit Test and Diagnosis under Process Variation using VTransform Coefficients," in Proc. 29th IEEE VLSI Test Symp., May 2011, pp. 64-69.

[24] S. Sindia, V. D. Agrawal, and V. Singh, "Test and Diagnosis of Analog Circuits Using Moment Generating Functions," in 20th IEEE Asian Test Symposium, 2011, pp. 371-376.

[25] S. Sindia, V. D. Agrawal, and V. Singh, "Testing Linear and Non-linear Analog Circuits using Moment Generating Functions," in Proc. 12th IEEE Latin American Test Workshop, Mar. 2011, pp. 1-6.

[26] S. Sindia, V. D. Agrawal, and V. Singh, "Defect Level and Fault Coverage in Coefficient Based Analog Circuit Testing," Journal of Electronic Testing: Theory and Applications, vol. 28, no. 4, pp. 541-549, Aug. 2012.

[27] S. Sindia, V. D. Agrawal, and V. Singh, "Parametric Fault Testing of Non-Linear Analog Circuits Based on Polynomial and V-Transform Coefficients," Journal of Electronic Testing: Theory and Applications, vol. 28, no. 5, pp. 757-771, Oct. 2012.

[28] S. Sindia, V. Singh, and V. D. Agrawal, "Bounds on Defect Level and Fault Coverage in Linear Analog Circuit Testing," in Proc. 13th VLSI Design and Test Symposium, July 2009, pp. 410-421.

[29] S. Sindia, V. Singh, and V. D. Agrawal, "Multi-Tone Testing of Linear and Nonlinear Analog Circuits Using Polynomial Coefficients," in Proc. 18th IEEE Asian Test Symposium, Nov. 2009, pp. 63-68.

[30] S. Sindia, V. Singh, and V. D. Agrawal, "Polynomial Coefficient Based DC Testing of Non-Linear Analog Circuits," in Proc. 19th ACM Great Lakes Symp. on VLSI, May 2009, pp. 69-74.

[31] S. Sindia, V. Singh, and V. D. Agrawal, "Polynomial Coefficient Based Multi-Tone Testing of Analog Circuits," in Proc. North Atlantic Test Workshop, May 2009, pp. 9-18.

[32] S. Sindia, V. Singh, and V. D. Agrawal, "V-Transform: An Enhanced Polynomial Coefficient Based DC Test for Non-Linear Analog Circuits," in Proc. of 8th IEEE East West Design-Test Symp., Sept. 2009, pp. 283-286.

[33] S. Sindia, V. Singh, and V. D. Agrawal, "Parametric Fault Diagnosis of Nonlinear Analog Circuits Using Polynomial Coefficients," in Proc. 23rd International Conf. VLSI Design, Jan. 2010, pp. 288-293.

[34] M. Slamani and B. Kaminska, "Analog Circuit Fault Diagnosis Based on Sensitivity Computation and Functional Testing," IEEE Design \& Test of Computers, vol. 19, no. 1, pp. 30-39, 1992.

[35] H.-G. Stratigopoulos and Y. Makris, "Error Moderation in Low-Cost Machine-Learning-Based Analog/RF Testing," IEEE Transactions on Computer-Aided Design, vol. 27, no. 2, pp. 339351, Feb. 2008.

[36] H.-G. Stratigopoulos and S. Mir, "Adaptive Alternate Analog Test," IEEE Design \& Test of Computers, vol. 29, no. 4, pp. 71-79, 2012.

[37] P. N. Variyam, S. Cherubal, and A. Chatterjee, "Prediction of Analog Performance Parameters Using Fast Transient Testing," IEEE Transactions on Computer-Aided Design, vol. 21, no. 3, pp. 349-361, Mar. 2002. 